\title{
INTUITIONISTIC FUZZY NORMAL SUBRINGS OVER NORMED RINGS
}

\author{
NOUR ABED ALHALEEM*, ABD GHAFUR AHMAD \\ Department of Mathematical Sciences, Faculty of Science and Technology, Universiti Kebangsaan Malaysia, \\ Bangi 43600, Selangor, Malaysia \\ *Corresponding author: noorb@ymail.com
}

\begin{abstract}
This paper presents the notion of intuitionistic fuzzy normed normal subrings. We investigate the concept of intuitionistic fuzzy normal subrings over normed rings and characterize relevant properties of such subrings. Further, we define direct product of fuzzy normal subrings over normed rings and investigate some related fundamental properties.
\end{abstract}

\section{INTRODUCTION}

After an introduction of fuzzy sets by Zadeh [1] several researchers investigated on the generalization of the concept of fuzzy set. In 1971, Rosenfeld [2] initiated the studies of fuzzy group theory by introducing the concepts of fuzzy subgroupoid and fuzzy subgroup. Later in 1981 [3], Wu introduced the notion of fuzzy normal subgroups of an ordinary group and Liu [4] defined a fuzzy invariant (normal) subgroup, Liu also extended the notion of a subring of a ring and the product of complexes to the fuzzy setting in the same paper. In 1984 [5], Mukharjee and Bhattacharya introduced the concept of fuzzy cosets and studied their relation with normal fuzzy subgroups, proved that for a group $G$ a fuzzy subgroup is fuzzy normal if and only if it is constant on the conjugate classes of $G$ and showed that the level subgroups of a fuzzy normal subgroup are all normal. Moreover, Wu in [6] introduced the concept of a normal fuzzy subgroup of a fuzzy group and used this concept to formulate the quotient structure of a fuzzy group. In [7], Mishref defined the

Received February $1^{\text {st }}, 2021$; accepted March $3^{\text {rd }}, 2021$; published April $1^{\text {st }}, 2021$.

2010 Mathematics Subject Classification. 03F55, 03E72.

Key words and phrases. intuitionistic fuzzy normed subrings; intuitionistic fuzzy normed normal subrings; direct product of intuitionistic fuzzy normed normal subrings.

(C)2021 Authors retain the copyrights of their papers, and all open access articles are distributed under the terms of the Creative Commons Attribution License. 
maximal normal fuzzy subgroup and gave some of its properties in analogy to the crisp case, also he defined the subnormal, normal and composition series of normal fuzzy subgroups and explained the interrelationship between them and the series in the crisp case. In [8], a new type of fuzzy normal subgroups and fuzzy cosets was presented.

The notion of intuitionistic fuzzy set was introduced by Atanassov [9] as a generalization of fuzzy sets. After that many researches applied this notion in various branches of mathematics especially in algebra and defined the notions of intuitionistic fuzzy subgroups, intuitionistic fuzzy subrings and intuitionistic fuzzy normal subgroups. Hur et al in [10], studied and characterized some properties of intuitionistic fuzzy normal subgroups of a group. In [11], Marashdeh and Salleh studied intuitionistic fuzzy groups by generalizing the notion of the fuzzy normal subgroup to the intuitionistic fuzzy normal subgroup. Later, some properties of intuitionistic fuzzy normal subrings were studied by Veeramani in [12], he described the algebraic nature of intuitionistic fuzzy normal subrings of a ring under homomorphism and anti-homomorphism. In [13], Sharma defined intuitionistic fuzzy magnified translation of intuitionistic fuzzy (normal) subring and ideal of a ring.

The notion of intuitionistic fuzzy normed rings was introduced by Abed Alhaleem and Ahmad in [14]. The concepts of intuitionistic fuzzy normed subrings and intuitionistic fuzzy normed ideals was presented as an extension of fuzzy normed rings which were studied by Emniyet and Sahin in [15], which presented the notions of fuzzy normed subrings and fuzzy normed ideals. A generalization of normed ring was studied by Aren in [16]. Later, Naimark introduced normed rings in [17], which gave the first comprehensive treatment of Banach algebras. Gel'fand defined commutative normed rings in [18] and addressed them as complex Banach spaces with introduction of the notion of commutative normed rings.

In this paper, we introduce the notion of intuitionistic fuzzy normed normal subrings. We study the algebraic nature of intuitionistic fuzzy normed normal subrings and characterize relevant properties. We introduce and study the notions of direct product of intuitionistic fuzzy normal subrings over normed rings. Further we define the relations between the intuitionistic characteristic function and intuitionistic fuzzy normed normal subrings.

\section{Preliminaries}

In this section, we outline the most significant definitions and results needed for the following sections. We review some basic ideas of intuitionistic fuzzy set, intuitionistic fuzzy normed subring, definitions of normed linear spaces, t-norm and s-norm. 
Definition 2.1. [19] The fuzzy set $A$ in a universal $X$ is a set of ordered pairs:

$$
A=\left\{\left(v, \mu_{A}(v)\right): v \in X\right\}
$$

Where, $\mu_{A}(v)$ is the membership function of $v$ in $A$ which associates each element in $X$ with a real number in the interval $[0,1]$.

Definition 2.2. [20] An intuitionistic fuzzy set (briefly, IFS) $A$ in a nonempty set $X$ is an object having the form IFS $A=\left\{\left(v, \mu_{A}(v), \gamma_{A}(v): v \in X\right\}\right.$, where the functions $\mu_{A}(v): X \rightarrow[0,1]$ and $\gamma_{A}(v): X \rightarrow[0,1]$ denote the degree of membership and the degree of nonmembership, respectively, where $\left.0 \leq \mu_{A}(v)+\gamma_{A}(v)\right) \leq 1$ for all $v \in X$. An intuitionistic fuzzy set $A$ is written symbolically in the form $A=\left(\mu_{A}, \gamma_{A}\right)$.

Definition 2.3. [17] $A$ ring $A$ is said to be a normed ring (NR) if $A$ possesses a norm \|\| , that is, a non-negative real-valued function \|\|$: A \rightarrow R$ such that for any $v, r \in A$,

(1) $\|v\|=0 \Leftrightarrow v=0$,

(2) $\|v+r\| \leq\|v\|+\|r\|$,

(3) $\|v\|=\|-v\|$, and

(4) $\|v r\| \leq\|v\|\|r\|$.

Definition 2.4. [14] Let $A=\left\{\left(v, \mu_{A}(v), \gamma_{A}(v)\right): v \in N R\right\}$ and $B=\left\{\left(v, \mu_{B}(v), \gamma_{B}(v)\right): v \in N R\right\}$ be two intuitionistic fuzzy normed rings over the normed ring NR. Then $A$ is an intuitionistic fuzzy normed subring of $B$ if $\mu_{A}(v) \leq \mu_{B}(v)$ and $\gamma_{A}(v) \geq \gamma_{B}(v)$.

Proposition 2.5. [14] Let $A$ be an intuitionistic fuzzy normed ring and let 0 be the zero of the normed ring $N R$, then the following is true for every $v \in N R$ :

(i) $\mu_{A}(v) \leq \mu_{A}(0), \gamma_{A}(0) \leq \gamma_{A}(v)$,

(ii) $\mu_{A}(v)=\mu_{A}(-v), \gamma_{A}(v)=\gamma_{A}(-v)$.

Lemma 2.6. [14] Let $1_{N R}$ be the multiplicative identity of $N R$ then for all $v \in N R$ :

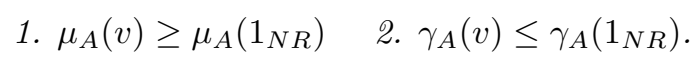

Proposition 2.7. [21] Let $A$ be an intuitionistic fuzzy set in a ring $R$, we denote the $(\alpha, \beta)$-cut set by $A_{\alpha, \beta}=\left\{v \in R: \mu_{A} \geq \alpha\right.$ and $\left.\gamma_{A} \leq \beta\right\}$ where $\alpha+\beta \leq 1$ and $\alpha, \beta \in[0,1]$.

Definition 2.8. [22] Let $*:[0,1] \times[0,1] \rightarrow[0,1]$ be a binary operation. Then $*$ is a $t$-norm if $*$ satisfies the conditions of commutativity, associativity, monotonicity and neutral element 1.

We shortly use $t$-norm and write $v * r$ instead of $*(v, r)$. Some examples of $t$-norm are $v * r=\min \{v, r\}$ and $v * r=v \cdot r$. 
Definition 2.9. [23] Let $\diamond:[0,1] \times[0,1] \rightarrow[0,1]$ be a binary operation. Then $\diamond$ is a s-norm if $\diamond$ satisfies the conditions of commutativity, associativity, monotonicity and neutral element 0.

We shortly use $s$-norm and write $v \diamond r$ instead of $\diamond(v, r)$. Some examples of $s$-norm are $v \diamond r=$ max $\{v, r\}$ and $v \diamond r=v+r-v \times r$.

\section{Some Properties of Intuitionistic Fuzzy Normed Normal Subrings}

Throughout the rest of this paper, $\mathbb{R}$ is the set of real numbers and $N R$ is a normed ring. We define the intuitionistic fuzzy normed normal subrings and some basic properties related to it.

Definition 3.1. [14] Let $*$ be a continuous t-norm and $\diamond$ be a continuous s-norm. An intuitionistic fuzzy set $A=\left\{\left(v, \mu_{A}(v), \gamma_{A}(v)\right): v \in N R\right\}$ is called an intuitionistic fuzzy normed subring (IFNSR) of the normed ring $(N R,+,$.$) if it satisfies the following conditions for all v, r \in N R$ :

(i) $\mu_{A}(v-r) \geq \mu_{A}(v) * \mu_{A}(r)$,

(ii) $\mu_{A}(v r) \geq \mu_{A}(v) * \mu_{A}(r)$,

(iii) $\gamma_{A}(v-r) \leq \gamma_{A}(v) \diamond \gamma_{A}(r)$,

(iv) $\gamma_{A}(v r) \leq \gamma_{A}(v) \diamond \gamma_{A}(r)$.

Definition 3.2. Let $N R$ be a normed ring. An intuitionistic fuzzy subring $A$ of $N R$ is said to be an intuitionistic fuzzy normed normal subring (IFNNSR) of NR if it satisfies the following for all $v, r \in R$ :

(i) $\mu_{A}(v r)=\mu_{A}(r v)$,

(ii) $\gamma_{A}(v r)=\gamma_{A}(r v)$

Proposition 3.3. Let $(N R,+,$.$) be a ring. If A$ and $B$ are two intuitionistic fuzzy normed normal subrings of $N R$, then their intersection $(A \cap B)$ is an intuitionistic fuzzy normed normal subring of $N R$.

Proof. Let $v, r \in N R$. Let $A=\left\{\left(v, \mu_{A}(v), \gamma_{A}(v)\right): v \in N R\right\}$ and $B=\left\{\left(v, \mu_{B}(v), \gamma_{B}(v)\right): v \in N R\right\}$ be intuitionistic fuzzy normed normal subrings. Let $C=A \cap B$ such that $C=\left\{\left(v, \mu_{C}(v), \gamma_{C}(v)\right): v \in N R\right\}$ where $\mu_{C}(v)=\min \left\{\mu_{A}(v), \mu_{B}(v)\right\}$ and $\gamma_{C}(v)=\max \left\{\gamma_{A}(v), \gamma_{B}(v)\right\}$.

$$
\begin{aligned}
\mu_{C}(v-r) & =\min \left\{\mu_{A}(v-r), \mu_{B}(v-r)\right\} \\
& =\mu_{A}(v-r) * \mu_{B}(v-r) \\
& \geq\left\{\mu_{A}(v) * \mu_{A}(r)\right\} *\left\{\mu_{B}(v) * \mu_{B}(r)\right\} \\
& =\mu_{A}(v) *\left\{\mu_{A}(r) * \mu_{B}(v)\right\} * \mu_{B}(r) \\
& =\mu_{A}(v) *\left\{\mu_{B}(v) * \mu_{A}(r)\right\} * \mu_{B}(r) \\
& =\left\{\mu_{A}(v) * \mu_{B}(v)\right\} *\left\{\mu_{A}(r) * \mu_{B}(r)\right\} \\
& =\mu_{C}(v) * \mu_{C}(r)
\end{aligned}
$$


and

$$
\begin{aligned}
\mu_{C}(v r) & =\min \left\{\mu_{A}(v r), \mu_{B}(v r)\right\} \\
& =\mu_{A}(v r) * \mu_{B}(v r) \\
& \geq\left\{\mu_{A}(v) * \mu_{A}(r)\right\} *\left\{\mu_{B}(v) * \mu_{B}(r)\right\} \\
& =\mu_{A}(v) *\left\{\mu_{A}(r) * \mu_{B}(v)\right\} * \mu_{B}(r) \\
& =\mu_{A}(v) *\left\{\mu_{B}(v) * \mu_{A}(r)\right\} * \mu_{B}(r) \\
& =\left\{\mu_{A}(v) * \mu_{B}(v)\right\} *\left\{\mu_{A}(r) * \mu_{B}(r)\right\} \\
& =\mu_{C}(v) * \mu_{C}(r) .
\end{aligned}
$$

Similarly

$$
\gamma_{C}(v-r) \leq \gamma_{C}(v) \diamond \gamma_{C}(r)
$$

and

$$
\gamma_{C}(v r) \leq \gamma_{C}(v) \diamond \gamma_{C}(r)
$$

Thus $C$ is an intuitionistic fuzzy normed subring of $N R$. Now,

$$
\begin{aligned}
\mu_{C}(v r) & =\mu_{A}(v r) * \mu_{B}(v r) \\
& =\mu_{A}(r v) * \mu_{B}(r v) \\
& =\mu_{C}(r v) .
\end{aligned}
$$

Therefore $\mu_{C}(v r)=\mu_{C}(r v)$.

Also

$$
\begin{aligned}
\gamma_{C}(v r) & =\gamma_{A}(v r) \diamond \gamma_{B}(v r) \\
& =\gamma_{A}(r v) \diamond \gamma_{B}(r v) \\
& =\gamma_{C}(r v) .
\end{aligned}
$$

Therefore $\gamma_{C}(v r)=\gamma_{C}(r v)$.

Then, the intersection of any two intuitionistic fuzzy normed normal subrings is an intuitionistic fuzzy normed normal subring of $N R$.

Definition 3.4. Let $A$ be a non-empty subset of the normed ring $N R$, the intuitionistic characteristic function of $A$ is defined as $\lambda_{A}=\left(\mu_{\lambda_{A}}, \gamma_{\lambda_{A}}\right)$, where

$\mu_{\lambda_{A}}(r)=\left\{\begin{array}{lll}1 & \text { if } & r \in A \\ 0, & \text { if } & r \notin A\end{array}, \gamma_{\lambda_{A}}(r)=\left\{\begin{array}{lll}0 & \text { if } & r \in A \\ 1, & \text { if } & r \notin A\end{array}\right.\right.$

Lemma 3.5. If $A=\left(\mu_{A}, \gamma_{A}\right)$ is a subring of $N R$ then $\lambda_{A}=\left(\mu_{\lambda_{A}}, \gamma_{\lambda_{A}}\right)$ is an intuitionistic fuzzy normed normal subring of $N R$. 
Proof. It shown in [14] that $\lambda_{A}=\left(\mu_{\lambda_{A}}, \gamma_{\lambda_{A}}\right)$ is an intuitionistic fuzzy normed subring of $N R$ when $A$ is a subring.

Now, we need to show that $\lambda_{A}=\left(\mu_{\lambda_{A}}, \gamma_{\lambda_{A}}\right)$ is an intuitionistic fuzzy normed normal subring, since $v r$ and $r v$ are in $A$, it follows that $\mu_{\lambda_{A}}(v r)=1=\mu_{\lambda_{A}}(r v)$ and $\gamma_{\lambda_{A}}(v r)=0=\gamma_{\lambda_{A}}(r v)$. Consequently, $\mu_{\lambda_{A}}(v r)=\mu_{\lambda_{A}}(r v)$ and $\gamma_{\lambda_{A}}(v r)=\gamma_{\lambda_{A}}(r v)$.

Thus the intuitionistic characteristic function $\lambda_{A}=\left(\mu_{\lambda_{A}}, \gamma_{\lambda_{A}}\right)$ of $A$ is an intuitionistic fuzzy normed normal subring of $N R$.

Lemma 3.6. If $A$ and $B$ are two subrings of the ring $N R$, then their intersection $A \cap B$ is a subring of $N R$ if and only if the intuitionistic characteristic function $\lambda_{C}=\left(\mu_{\lambda_{C}}, \gamma_{\lambda_{C}}\right)$ of $C=A \cap B$ is an intuitionistic fuzzy normed normal subring of $N R$.

Proof. Let $C=A \cap B$ be a subring of $N R$ and $v, r \in N R$. If $v, r \in C$, then by definition of intuitionistic characteristic function $\mu_{\lambda_{C}}(v)=1=\mu_{\lambda_{C}}(r)$ and $\gamma_{\lambda_{C}}(v)=0=\gamma_{\lambda_{C}}(r)$. Since $v-r$, vr in $A$ and $B$, it follows that $v-r$, vr in $C$. Thus, $\mu_{\lambda_{C}}(r-v)=1=1 * 1=\mu_{\lambda_{C}}(r) * \mu_{\lambda_{C}}(v)$ and $\mu_{\lambda_{C}}(r v)=1=1 * 1=\mu_{\lambda_{C}}(r) * \mu_{\lambda_{C}}(v)$. Thus $\mu_{\lambda_{C}}(r-v) \geq \mu_{\lambda_{C}}(r) * \mu_{\lambda_{C}}(v)$ and $\mu_{\lambda_{C}}(r v) \geq \mu_{\lambda_{C}}(r) * \mu_{\lambda_{C}}(v)$. Now $\gamma_{\lambda_{C}}(v-r)=0=0 \diamond 0=$ $\gamma_{\lambda_{C}}(v) \diamond \gamma_{\lambda_{C}}(r)$ and $\gamma_{\lambda_{C}}(v r)=0=0 \diamond 0=\gamma_{\lambda_{C}}(v) \diamond \gamma_{\lambda_{C}}(r)$. Thus, $\gamma_{\lambda_{C}}(v-r) \leq \gamma_{\lambda_{C}}(v) \diamond \gamma_{\lambda_{C}}(r)$ and $\gamma_{\lambda_{C}}(v r) \leq \gamma_{\lambda_{C}}(v) \diamond \gamma_{\lambda_{C}}(r)$. As $v r$ and $r v \in C$, so $\mu_{\lambda_{C}}(r v)=1=\mu_{\lambda_{C}}(v r)$ and $\gamma_{\lambda_{C}}(v r)=0=\gamma_{\lambda_{C}}(r v)$. Accordingly, $\mu_{\lambda_{C}}(r v)=\mu_{\lambda_{C}}(v r)$ and $\gamma_{\lambda_{C}}(v r)=\gamma_{\lambda_{C}}(r v)$. Similarly we have when $v, r \notin C$ :

$$
\begin{array}{ccc}
\mu_{\lambda_{C}}(v-r) \geq \mu_{\lambda_{C}}(v) * \mu_{\lambda_{C}}(r) & \text { and } & \mu_{\lambda_{C}}(v r) \geq \mu_{\lambda_{C}}(v) * \mu_{\lambda_{C}}(r), \\
\gamma_{\lambda_{C}}(v-r) \leq \gamma_{\lambda_{C}}(v) \diamond \gamma_{\lambda_{C}}(r) & \text { and } & \gamma_{\lambda_{C}}(v r) \leq \gamma_{\lambda_{C}}(v) \diamond \gamma_{\lambda_{C}}(r), \\
\mu_{\lambda_{C}}(v r)=\mu_{\lambda_{C}}(r v) & \text { and } & \gamma_{\lambda_{C}}(v r)=\gamma_{\lambda_{C}}(r v) .
\end{array}
$$

Hence the intuitionistic characteristic function $\lambda_{C}=\left(\mu_{\lambda_{C}}, \gamma_{\lambda_{C}}\right)$ of $C$ is an intuitionistic fuzzy normed normal subring of $N R$.

Conversely, assume that the intuitionistic characteristic function $\lambda_{C}=\left(\mu_{\lambda_{C}}, \gamma_{\lambda_{C}}\right)$ of $C$ is an intuitionistic fuzzy normal normed subring of $N R$. Let $v, r \in C$, this imply that $\mu_{\lambda_{C}}(v)=1=\mu_{\lambda_{C}}(r)$ and $\gamma_{\lambda_{C}}(v)=0=$ $\gamma_{\lambda_{C}}(r)$, then:

$$
\begin{gathered}
\mu_{\lambda_{C}}(v-r) \quad \geq \mu_{\lambda_{C}}(v) * \mu_{\lambda_{C}}(r)=1 * 1=1, \\
\mu_{\lambda_{C}}(v r) \quad \geq \mu_{\lambda_{C}}(v) * \mu_{\lambda_{C}}(r)=1 * 1=1, \\
\gamma_{\lambda_{C}}(v-r) \quad \leq \gamma_{\lambda_{C}}(v) \diamond \gamma_{\lambda_{C}}(r)=0 \diamond 0=0, \\
\gamma_{\lambda_{C}}(v r) \quad \leq \gamma_{\lambda_{C}}(v) \diamond \gamma_{\lambda_{C}}(r) \quad=0 \diamond 0=0 .
\end{gathered}
$$

This implies that $\mu_{\lambda_{C}}(v-r)=1, \mu_{\lambda_{C}}(v r)=1$ and $\gamma_{\lambda_{C}}(v-r)=0, \gamma_{\lambda_{C}}(v r)=0$. Thus, $v-r$ and $v r \in C$. Hence $C$ is a subring of $N R$.

Proposition 3.7. If $A$ is an intuitionistic fuzzy normed normal subring of a ring $N R$. Then $\triangle A=\left(\mu_{A}, \mu_{A}^{c}\right)$ is an intuitionistic fuzzy normed normal subring of a ring $N R$. 
Proof. Let $v, r \in N R$

$$
\begin{aligned}
\mu_{A}^{c}(v-r) & =1-\mu_{A}(v-r) \\
& \leq 1-\left(\mu_{A}(v) * \mu_{A}(r)\right) \\
& \leq 1-\min \left\{\mu_{A}(v), \mu_{A}(r)\right\} \\
& =\max \left\{1-\mu_{A}(v), 1-\mu_{A}(r)\right\} \\
& =\max \left\{\mu_{A}^{c}(v), \mu_{A}^{c}(r)\right\} .
\end{aligned}
$$

Then, $\mu_{A}^{c}(v-r) \leq \mu_{A}^{c}(v) \diamond \mu_{A}^{c}(r)$.

$$
\begin{aligned}
\mu_{A}^{c}(v r) & =1-\mu_{A}(v r) \\
& \leq 1-\left(\mu_{A}(v) * \mu_{A}(r)\right) \\
& \leq 1-\min \left\{\mu_{A}(v), \mu_{A}(r)\right\} \\
& =\max \left\{1-\mu_{A}(v), 1-\mu_{A}(r)\right\} \\
& =\max \left\{\mu_{A}^{c}(v), \mu_{A}^{c}(r)\right\}
\end{aligned}
$$

Then, $\mu_{A}^{c}(v r) \leq \mu_{A}^{c}(v) \diamond \mu_{A}^{c}(r)$.

Also, $\mu_{A}^{c}(v r)=1-\mu_{A}(v r)=1-\mu_{A}(r v)=\mu_{A}^{c}(r v)$, then $\mu_{A}^{c}(v r)=\mu_{A}^{c}(r v)$.

Therefore, $\triangle A=\left(\mu_{A}, \mu_{A}^{c}\right)$ is an intuitionistic fuzzy normed normal subring of $N R$.

Proposition 3.8. If $A$ is an intuitionistic fuzzy normed normal subring of a ring $N R$. Then $\diamond A=\left(\gamma_{A}^{c}, \gamma_{A}\right)$ is an intuitionistic fuzzy normed normal subring of a ring $N R$.

Proof. Let $v, r \in N R$

$$
\begin{aligned}
\gamma_{A}^{c}(v-r) & =1-\gamma_{A}(v-r) \\
& \geq 1-\left(\gamma_{A}(v) \diamond \gamma_{A}(r)\right) \\
& \geq 1-\max \left\{\gamma_{A}(v), \gamma_{A}(r)\right\} \\
& =\min \left\{1-\gamma_{A}(v), 1-\gamma_{A}(r)\right\} \\
& =\min \left\{\gamma_{A}^{c}(v), \gamma_{A}^{c}(r)\right\}
\end{aligned}
$$

Then, $\gamma_{A}^{c}(v-r) \geq \gamma_{A}^{c}(v) * \gamma_{A}^{c}(r)$.

$$
\begin{aligned}
\gamma_{A}^{c}(v r) & =1-\gamma_{A}(v r) \\
& \geq 1-\left(\gamma_{A}(v) \diamond \gamma_{A}(r)\right) \\
& \geq 1-\max \left\{\gamma_{A}(v), \gamma_{A}(r)\right\} \\
& =\min \left\{1-\mu_{A}(v), 1-\gamma_{A}(r)\right\} \\
& =\min \left\{\gamma_{A}^{c}(v), \gamma_{A}^{c}(r)\right\}
\end{aligned}
$$

Then, $\gamma_{A}^{c}(v r) \geq \gamma_{A}^{c}(v) * \gamma_{A}^{c}(r)$.

Also, $\gamma_{A}^{c}(v r)=1-\gamma_{A}(v r)=1-\gamma_{A}(r v)=\gamma_{A}^{c}(r v)$, then $\gamma_{A}^{c}(v r)=\gamma_{A}^{c}(r v)$.

Therefore, $\diamond A=\left(\gamma_{A}^{c}, \gamma_{A}\right)$ is an intuitionistic fuzzy normed normal ideal of $N R$. 
Proposition 3.9. If $A$ is an intuitionistic fuzzy normed normal subring of a ring $N R$. Then $A=\left(\mu_{A}, \gamma_{A}\right)$ is an intuitionistic fuzzy normed normal subring of $N R$ if the fuzzy subsets $\mu_{A}$ and $\gamma_{A}^{c}$ are intuitionistic fuzzy normed normal subrings of $N R$.

Proof. Clearly, $\mu_{A}$ is an intuitionistic fuzzy normed normal subring of $N R$, we need to show that $\gamma_{A}$ is an intuitionistic fuzzy normed normal subring of $N R$.

$$
\begin{aligned}
1-\gamma_{A}(v-r) & =\gamma_{A}^{c}(v-r) \\
& \geq \gamma_{A}^{c}(v) * \gamma_{A}^{c}(r) \\
& \geq \min \left\{\gamma_{A}^{c}(v), \gamma_{A}^{c}(r)\right\} \\
& =\min \left\{1-\gamma_{A}(v), 1-\gamma_{A}(r)\right\} \\
& =1-\max \left\{\gamma_{A}(v), \gamma_{A}(r)\right\} .
\end{aligned}
$$

Then, $\gamma_{A}(v-r) \leq \gamma_{A}(v) \diamond \gamma_{A}(r)$.

$$
\begin{aligned}
1-\gamma_{A}(v r) & =\gamma_{A}^{c}(v r) \\
& \geq \gamma_{A}^{c}(v) * \gamma_{A}^{c}(r) \\
& \geq \min \left\{\gamma_{A}^{c}(v), \gamma_{A}^{c}(r)\right\} \\
& =\min \left\{1-\gamma_{A}(v), 1-\gamma_{A}(r)\right\} \\
& =1-\max \left\{\gamma_{A}(v), \gamma_{A}(r)\right\}
\end{aligned}
$$

Then, $\gamma_{A}(v r) \leq \gamma_{A}(v) \diamond \gamma_{A}(r)$.

Also, $1-\gamma_{A}(v r)=\gamma_{A}^{c}(v r)=\gamma_{A}^{c}(r v)=1-\gamma_{A}(r v)$. Then, $\gamma_{A}(v r)=\gamma_{A}(r v)$.

Hence, $A=\left(\mu_{A}, \gamma_{A}\right)$ is an intuitionistic fuzzy normed normal subring of $N R$.

Proposition 3.10. If $A$ is an intuitionistic fuzzy normed normal subring of a ring $N R$. Then $A=\left(\mu_{A}, \gamma_{A}\right)$ is an intuitionistic fuzzy normed normal subring of $N R$ if the fuzzy subsets $\mu_{A}^{c}$ and $\gamma_{A}$ are intuitionistic fuzzy normed normal subrings of $N R$.

Proof. Clearly, $\gamma_{A}$ is an intuitionistic fuzzy normed normal subring of $N R$. We need to show that $\mu_{A}$ is an intuitionistic fuzzy normed normal subring of $N R$.

$$
\begin{aligned}
1-\mu_{A}(v-r) & =\mu_{A}^{c}(v-r) \\
& \leq \mu_{A}^{c}(v) \diamond \mu_{A}^{c}(r) \\
& \leq \max \left\{\mu_{A}^{c}(v), \mu_{A}^{c}(r)\right\} \\
& =\max \left\{1-\mu_{A}(v), 1-\mu_{A}(r)\right\} \\
& =1-\min \left\{\mu_{A}(v), \gamma_{A}(r)\right\} .
\end{aligned}
$$


Then, $\mu_{A}(v-r) \geq \mu_{A}(v) * \mu_{A}(r)$.

$$
\begin{aligned}
1-\mu_{A}(v r) & =\mu_{A}^{c}(v r) \\
& \leq \mu_{A}^{c}(v) \diamond \mu_{A}^{c}(r) \\
& \leq \max \left\{\mu_{A}^{c}(v), \mu_{A}^{c}(r)\right\} \\
& =\max \left\{1-\mu_{A}(v), 1-\mu_{A}(r)\right\} \\
& =1-\min \left\{\mu_{A}(v), \mu_{A}(r)\right\} .
\end{aligned}
$$

Then, $\mu_{A}(v r) \geq \mu_{A}(v) * \mu_{A}(r)$.

Also, $1-\mu_{A}(v r)=\mu_{A}^{c}(v r)=\mu_{A}^{c}(r v)=1-\mu_{A}(r v)$. Then, $\mu_{A}(v r)=\mu_{A}(r v)$

Hence, $A=\left(\mu_{A}, \gamma_{A}\right)$ is an intuitionistic fuzzy normed normal subring of $N R$.

\section{Direct PRoduct of INTUitionistic FUZZY NORMED NORMAL SUbRings}

In this section, we define the direct product of intuitionistic fuzzy sets $A_{1}, A_{2}$ of normed rings $R_{1}, R_{2}$, respectively and examine some fundamental properties of direct product of intuitionistic fuzzy normed normal subrings. If $N R_{1}, N R_{2}$ are normed rings, then the direct product $N R_{1} \times N R_{2}$ of $N R_{1}$ and $N R_{2}$ is a normed ring with addition + defined as $(v, r)+(z, d)=(v+z, r+d)$ and multiplication $\circ$ defined as $(v, r) \circ(z, d)=(v z, r d)$ for every $(v, r),(z, d)$ in $N R_{1} \times N R_{2}$.

Definition 4.1. An intuitionistic fuzzy set (IFS) $A \times B=\left(\mu_{A \times B}, \gamma_{A \times B}\right)$ of $N R_{1} \times N R_{2}$ is an intuitionistic fuzzy normed subring (IFNSR) of $N R_{1} \times N R_{2}$ if for all $v=\left(v_{1}, v_{2}\right)$ and $r=\left(r_{1}, r_{2}\right)$ in $N R_{1} \times N R_{2}$, satisfies:

(i) $\mu_{A \times B}(v-r) \geq \mu_{A \times B}(v) * \mu_{A \times B}(r)$;

(ii) $\mu_{A \times B}(v r) \geq \mu_{A \times B}(v) * \mu_{A \times B}(r)$;

(iii) $\gamma_{A \times B}(v-r) \leq \gamma_{A \times B}(v) \diamond \gamma_{A \times B}(r)$;

(iv) $\gamma_{A \times B}(v r) \leq \gamma_{A \times B}(v) \diamond \gamma_{A \times B}(r)$.

Definition 4.2. An intuitionistic fuzzy normed subring $A \times B=\left(\mu_{A \times B}, \gamma_{A \times B}\right)$ of ring $N R_{1} \times N R_{2}$ is an intuitionistic fuzzy normed normal subring of $R_{1} \times R_{2}$ if for all $v=\left(v_{1}, v_{2}\right)$ and $r=\left(r_{1}, r_{2}\right)$ in $R_{1} \times R_{2}$ :

$$
\mu_{A \times B}(v r)=\mu_{A \times B}(r v) \quad \text { and } \quad \gamma_{A \times B}(v r)=\gamma_{A \times B}(r v) .
$$

Lemma 4.3. If $A$ and $B$ are intuitionistic fuzzy normed subrings of the rings $N R_{1}$ and $N R_{2}$, respectively, then $A \times B$ is an intuitionistic fuzzy normed subring of the ring $N R_{1} \times N R_{2}$ under the same operations defined in $N R_{1} \times N R_{2}$.

Let $A$ and $B$ be two intuitionistic fuzzy normed subsets of $N R_{1}$ and $N R_{2}$, respectively. The direct product of $A$ and $B$, is denoted by $A \times B$, and defined as

$$
A \times B=\left\{\left((v, r), \mu_{A \times B}(v, r), \gamma_{A \times B}(v, r)\right): \text { for all } v \in N R_{1} \text { and } r \in N R_{2}\right\}
$$


where $\mu_{A \times B}(v, r)=\min \left\{\mu_{A}(v), \mu_{B}(r)\right\}$ and $\gamma_{A \times B}(v, r)=\max \left\{\gamma_{A}(v), \gamma_{B}(r)\right\}$.

Lemma 4.4. If $A$ and $B$ are intuitionistic fuzzy normed normal subrings of rings $N R_{1}$ and $N R_{2}$, respectively, then $A \times B$ is also an intuitionistic fuzzy normed normal subring $N R_{1} \times N R_{2}$.

Proof. Since the direct product of $A$ and $B$ is denoted by $A \times B=\left(\mu_{A \times B}, \gamma_{A \times B}\right)$. Let $(v, r),(z, d)$ be in $N R_{1} \times N R_{2}$, then:

$$
\begin{aligned}
\mu_{A \times B}((v, r)-(z, d)) & =\mu_{A \times B}(v-z, r-d) \\
& =\min \left\{\mu_{A}(v-z), \mu_{B}(r-d)\right\} \\
& =\mu_{A}(v-z) * \mu_{B}(r-d) \\
& \geq\left\{\mu_{A}(v) * \mu_{A}(z)\right\} *\left\{\mu_{B}(r) * \mu_{B}(d)\right\} \\
& =\mu_{A}(v) *\left\{\mu_{A}(z) * \mu_{B}(r)\right\} * \mu_{B}(d) \\
& =\mu_{A}(v) *\left\{\mu_{B}(r) * \mu_{A}(z)\right\} * \mu_{B}(d) \\
& =\left\{\mu_{A}(v) * \mu_{B}(r)\right\} *\left\{\mu_{A}(z) * \mu_{B}(d)\right\} \\
& =\mu_{A \times B}(v, r) * \mu_{A \times B}(z, d)
\end{aligned}
$$

and

$$
\begin{aligned}
\mu_{A \times B}((v, r) \circ(z, d)) & =\mu_{A \times B}(v z, r d) \\
& =\min \left\{\mu_{A}(v z), \mu_{B}(r d)\right\} \\
& =\mu_{A}(v z) * \mu_{B}(r d) \\
& \geq\left\{\mu_{A}(v) * \mu_{A}(z)\right\} *\left\{\mu_{B}(r) * \mu_{B}(d)\right\} \\
& =\mu_{A}(v) *\left\{\mu_{A}(z) * \mu_{B}(r)\right\} * \mu_{B}(d) \\
& =\mu_{A}(v) *\left\{\mu_{B}(r) * \mu_{A}(z)\right\} * \mu_{B}(d) \\
& =\left\{\mu_{A}(v) * \mu_{B}(r)\right\} *\left\{\mu_{A}(z) * \mu_{B}(d)\right\} \\
& =\mu_{A \times B}(v, r) * \mu_{A \times B}(z, d) .
\end{aligned}
$$

Therefore, $A \times B$ is an intuitionistic fuzzy normed subring of $N R_{1} \times N R_{2}$.

Now,

$$
\begin{aligned}
\mu_{A \times B}((v, r) \circ(z, d)) & =\mu_{A \times B}(v z, r d) \\
& =\min \left\{\mu_{A}(v z), \mu_{B}(r d)\right\} \\
& =\min \left\{\mu_{A}(z v), \mu_{B}(d r)\right\} \\
& =\mu_{A \times B}(z v, d r) \\
& =\mu_{A \times B}((z, d) \circ(v, r)) .
\end{aligned}
$$

Similarly,

$$
\begin{aligned}
& \gamma_{A \times B}((v, r)-(z, d)) \leq \gamma_{A \times B}(v, r) \diamond \gamma_{A \times B}(z, d), \\
& \gamma_{A \times B}((v, r) \circ(z, d)) \leq \gamma_{A \times B}(v, r) \diamond \gamma_{A \times B}(z, d) .
\end{aligned}
$$

and $\gamma_{A \times B}((v, r) \circ(z, d))=\gamma_{A \times B}((z, d) \circ(v, r))$.

Hence, $A \times B$ is an intuitionistic fuzzy normed normal subring of $N R_{1} \times N R_{2}$. 
Proposition 4.5. Let $A$ and $B$ be an intuitionistic fuzzy subsets of the rings $N R_{1}$ and $N R_{2}$ with identities $1_{N R_{1}}$ and $1_{N R_{2}}$, respectively. If $A \times B$ is an intuitionistic fuzzy normed subring of $N R_{1} \times N R_{2}$, then at least one of the following must holds:

(i) $\mu_{A}(v) \leq \mu_{B}\left(1_{N R_{2}}\right)$ and $\gamma_{A}(v) \geq \gamma_{B}\left(1_{N R_{2}}\right)$; for all $v \in N R_{1}$,

(ii) $\mu_{B}(r) \leq \mu_{A}\left(1_{N R_{1}}\right)$ and $\gamma_{B}(r) \geq \gamma_{A}\left(1_{N R_{1}}\right)$; for all $r \in N R_{2}$.

Proof. Let $A \times B$ be an intuitionistic fuzzy normed subring of $N R_{1} \times N R_{2}$, and let the statements (i) and (ii) does not holds, we can find $v \in N R_{1}$ and $r \in N R_{2}$ such that $\mu_{A}(v)>\mu_{B}\left(1_{N R_{2}}\right), \gamma_{A}(v)<\gamma_{B}\left(1_{N R_{2}}\right)$ and $\mu_{B}(r)>\mu_{A}\left(1_{N R_{1}}\right), \gamma_{B}(r)<\gamma_{A}\left(1_{N R_{1}}\right)$. Thus we have

$$
\begin{aligned}
\mu_{A \times B}(v r) & =\min \left\{\mu_{A}(v), \mu_{B}(r)\right\} \\
& >\min \left\{\mu_{A}\left(1_{N R_{1}}\right), \mu_{B}\left(1_{N R_{2}}\right)\right\} \\
& =\mu_{A \times B}\left(1_{N R_{1}}, 1_{N R_{2}}\right)
\end{aligned}
$$

and

$$
\begin{aligned}
\gamma_{A \times B}(v r) & =\max \left\{\gamma_{A}(v), \gamma_{B}(r)\right\} \\
& <\max \left\{\gamma_{A}\left(1_{N R_{1}}\right), \gamma_{B}\left(1_{N R_{2}}\right)\right\} \\
& =\gamma_{A \times B}\left(1_{N R_{1}}, 1_{N R_{2}}\right)
\end{aligned}
$$

which implies that $A \times B$ is not an intuitionistic fuzzy normed subring of $N R_{1} \times N R_{2}$ which a contradiction. Therefore, at least one of the statements must hold.

Lemma 4.6. Let $A$ and $B$ be an intuitionistic fuzzy subsets of the rings $N R_{1}$ and $N R_{2}$ with identities $1_{N R_{1}}$ and $1_{N R_{2}}$, respectively. If $A \times B$ is an intuitionistic fuzzy normed normal subring of $N R_{1} \times N R_{2}$, then the following are true:

(i) if $\mu_{A}(v) \leq \mu_{B}\left(1_{N R_{2}}\right)$ and $\gamma_{A}(v) \geq \gamma_{B}\left(1_{N R_{2}}\right)$, then $A$ is an intuitionistic fuzzy normed normal subring of $N R_{1}$.

(ii) if $\mu_{B}(v) \leq \mu_{A}\left(1_{N R_{1}}\right)$ and $\gamma_{B}(v) \geq \gamma_{A}\left(1_{N R_{1}}\right)$, then $B$ is an intuitionistic fuzzy normed normal subring of $\mathrm{NR}_{2}$.

Proof. Let $A \times B$ be an intuitionistic fuzzy normed normal subring of $N R_{1} \times N R_{2}$ with $v, r \in N R_{1}$ and $1_{N R_{2}} \in N R_{2}$. Then $\left(v, 1_{N R_{2}}\right)$ and $\left(r, 1_{N R_{2}}\right)$ are in $N R_{1} \times N R_{2}$. Obviously, $A$ is an intuitionistic fuzzy normed subring of $N R_{1}$, then 
i.

$$
\begin{aligned}
\mu_{A}(v-r) & =\mu_{A}(v+(-r))=\min \left\{\mu_{A}(v+(-r)), \mu_{B}\left(1_{N R_{2}}+\left(-1_{N R_{2}}\right)\right)\right\} \\
& =\mu_{A \times B}\left((v+(-r)),\left(1_{N R_{2}}+\left(-1_{N R_{2}}\right)\right)\right. \\
& =\mu_{A \times B}\left(\left(v, 1_{N R_{2}}\right)+\left(-r,-1_{N R_{2}}\right)\right) \\
& =\mu_{A \times B}\left(\left(v, 1_{N R_{2}}\right)-\left(r, 1_{N R_{2}}\right)\right) \\
& \geq \mu_{A \times B}\left(v, 1_{N R_{2}}\right) * \mu_{A \times B}\left(r, 1_{N R_{2}}\right) \\
& =\min \left\{\mu_{A}(v), \mu_{B}\left(1_{N R_{2}}\right)\right\} * \min \left\{\mu_{A}(r), \mu_{B}\left(1_{N R_{2}}\right)\right\} \\
& =\mu_{A}(v) * \mu_{A}(r) .
\end{aligned}
$$

Also,

$$
\begin{aligned}
\mu_{A}(v r) & =\min \left\{\mu_{A}(v r), \mu_{B}\left(1_{N R_{2}} 1_{N R_{2}}\right)\right\} \\
& =\mu_{A \times B}\left(v r, 1_{N R_{2}} 1_{N R_{2}}\right) \\
& =\mu_{A \times B}\left(\left(v, 1_{N R_{2}}\right) \circ\left(r, 1_{N R_{2}}\right)\right) \\
& \geq \mu_{A \times B}\left(v, 1_{N R_{2}}\right) * \mu_{A \times B}\left(r, 1_{N R_{2}}\right) \\
& =\min \left\{\mu_{A}(v), \mu_{B}\left(1_{N R_{2}}\right)\right\} * \min \left\{\mu_{A}(r), \mu_{B}\left(1_{N R_{2}}\right)\right\} \\
& =\mu_{A}(v) * \mu_{A}(r)
\end{aligned}
$$

and with,

$$
\begin{aligned}
\mu_{A}(v r) & =\min \left\{\mu_{A}(v r), \mu_{B}\left(1_{N R_{2}} 1_{N R_{2}}\right)\right\} \\
& =\mu_{A \times B}\left((v r),\left(1_{N R_{2}} 1_{N R_{2}}\right)\right) \\
& =\mu_{A \times B}\left(\left(v, 1_{N R_{2}}\right) \circ\left(r, 1_{N R_{2}}\right)\right) \\
& =\mu_{A \times B}\left(\left(r, 1_{N R_{2}}\right) \circ\left(v, 1_{N R_{2}}\right)\right) \\
& =\mu_{A \times B}\left((r v),\left(1_{N R_{2}} 1_{N R_{2}}\right)\right) \\
& =\min \left\{\mu_{A}(r v), \mu_{B}\left(1_{N R_{2}} 1_{N R_{2}}\right)\right\} \\
& =\mu_{A}(r v) .
\end{aligned}
$$

Similarly, we can prove that $\gamma_{A}(v-r) \leq \gamma_{A}(v) \diamond \gamma_{A}(r), \gamma_{A}(v r) \leq \gamma_{A}(v) \diamond \gamma_{A}(r)$ and $\gamma_{A}(v r)=\gamma_{A}(r v)$ for all $v, r \in N R_{1}$. Hence, $A$ is an intuitionistic fuzzy normed normal subring of $N R_{1}$.

ii. The proof is similar to the above.

Definition 4.7. Let $A \times B$ be a non-empty subset of the ring $N R_{1} \times N R_{2}$. The intuitionistic characteristic function of $A \times B$ is denoted by $\lambda_{A \times B}=\left(\mu_{\lambda_{A \times B}}, \gamma_{\lambda_{A \times B}}\right)$ and defined as:

$\mu_{\lambda_{A \times B}}(r)=\left\{\begin{array}{lll}1 & \text { if } & r \in A \times B \\ 0 & \text { if } & r \notin A \times B\end{array}, \gamma_{\lambda_{A \times B}}(r)=\left\{\begin{array}{lll}0 & \text { if } & r \in A \times B \\ 1 & \text { if } & r \notin A \times B\end{array}\right.\right.$

Theorem 4.8. Let $A$ and $B$ be two subrings of the rings $N R_{1}$ and $N R_{2}$, respectively. Then $A \times B$ is a subring of $N R_{1} \times N R_{2}$ if and only if the intuitionistic characteristic function $\lambda_{C}=\left(\mu_{\lambda_{C}}, \gamma_{\lambda_{C}}\right)$ of $C=A \times B$ is an intuitionistic fuzzy normed normal subring of $N R_{1} \times N R_{2}$. 
Proof. Let $C=A \times B$ be a subring of $N R_{1} \times N R_{2}$ and $v, r \in N R_{1} \times N R_{2}$. If $v, r \in C=A \times B$, then by definition of intuitionistic characteristic function $\mu_{\lambda_{C}}(v)=1=\mu_{\lambda_{C}}(r)$ and $\gamma_{\lambda_{C}}(v)=0=\gamma_{\lambda_{C}}(r)$. Since $v-r$ and $v r \in C$ and $C$ is a subring. It follows that $\mu_{\lambda_{C}}(v-r)=1=1 * 1=\mu_{\lambda_{C}}(v) * \mu_{\lambda_{C}}(r)$ and $\mu_{\lambda_{C}}(v r)=1=1 * 1=\mu_{\lambda_{C}}(v) * \mu_{\lambda_{C}}(r)$. Thus $\mu_{\lambda_{C}}(v-r) \geq \mu_{\lambda_{C}}(v) * \mu_{\lambda_{C}}(r)$ and $\mu_{\lambda_{C}}(v r) \geq \mu_{\lambda_{C}}(v) * \mu_{\lambda_{C}}(r)$. Now $\gamma_{\lambda_{C}}(v-r)=0=0 \diamond 0=\gamma_{\lambda_{C}}(v) \diamond \gamma_{\lambda_{C}}(r)$ and $\gamma_{\lambda_{C}}(v-r)=0=0 \diamond 0=\gamma_{\lambda_{C}}(v) \diamond \gamma_{\lambda_{C}}(r)$. Thus $\gamma_{\lambda_{C}}(v-r) \leq \gamma_{\lambda_{C}}(v) \diamond \gamma_{\lambda_{C}}(r)$ and $\gamma_{\lambda_{C}}(v r) \leq \gamma_{\lambda_{C}}(v) \diamond \gamma_{\lambda_{C}}(r)$. As $v r$ and $r v \in C$, so $\mu_{\lambda_{C}}(v r)=1=\mu_{\lambda_{C}}(r v)$ and $\gamma_{\lambda_{C}}(v r)=0=\gamma_{\lambda_{C}}(r v)$. This implies that $\mu_{\lambda_{C}}(v r)=\mu_{\lambda_{C}}(r v)$ and $\gamma_{\lambda_{C}}(v r)=\gamma_{\lambda_{C}}(r v)$. Similarly we have

$$
\begin{array}{ccc}
\mu_{\lambda_{C}}(v-r) \geq \mu_{\lambda_{C}}(v) * \mu_{\lambda_{C}}(r) & \text { and } & \mu_{\lambda_{C}}(v r) \geq \mu_{\lambda_{C}}(v) * \mu_{\lambda_{C}}(r), \\
\gamma_{\lambda_{C}}(v-r) \leq \gamma_{\lambda_{C}}(v) \diamond \gamma_{\lambda_{C}}(r) & \text { and } & \gamma_{\lambda_{C}}(v r) \leq \gamma_{\lambda_{C}}(v) \diamond \gamma_{\lambda_{C}}(r), \\
\mu_{\lambda_{C}}(v r)=\mu_{\lambda_{C}}(r v) & \text { and } & \gamma_{\lambda_{C}}(v r)=\gamma_{\lambda_{C}}(r v) .
\end{array}
$$

when $v, r \notin C$. Hence the intuitionistic characteristic function $\lambda_{C}=\left(\mu_{\lambda_{C}}, \gamma_{\lambda_{C}}\right)$ of $C=A \times B$ is an intuitionistic fuzzy normed normal subring of $N R_{1} \times N R_{2}$.

On the other hand, assume that the intuitionistic characteristic function $\lambda_{C}=\left(\mu_{\lambda_{C}}, \gamma_{\lambda_{C}}\right)$ of $C=A \times B$ is an intuitionistic fuzzy normed normal subring of $N R_{1} \times N R_{2}$. Now we have to show that $C=A \times B$ is a subring of $N R$. Let $v, r \in C$, where $v=\left(v^{\prime}, r^{\prime}\right)$ and $r=\left(v^{\prime \prime}, r^{\prime \prime}\right)$, where $v^{\prime}, v^{\prime \prime} \in A$ and $r^{\prime}, r^{\prime \prime} \in B$. By definition $\mu_{\lambda_{C}}(v)=1=\mu_{\lambda_{C}}(r)$ and $\gamma_{\lambda_{C}}(v)=0=\gamma_{\lambda_{C}}(r)$,

$$
\begin{gathered}
\mu_{\lambda_{C}}(v-r) \quad \geq \mu_{\lambda_{C}}(v) * \mu_{\lambda_{C}}(r)=1 * 1=1, \\
\mu_{\lambda_{C}}(v r) \quad \geq \mu_{\lambda_{C}}(v) * \mu_{\lambda_{C}}(r)=1 * 1=1, \\
\gamma_{\lambda_{C}}(v-r) \quad \leq \gamma_{\lambda_{C}}(v) \diamond \gamma_{\lambda_{C}}(r)=0 \diamond 0=0, \\
\gamma_{\lambda_{C}}(v r) \quad \leq \gamma_{\lambda_{C}}(v) \diamond \gamma_{\lambda_{C}}(r) \quad=0 \diamond 0=0 .
\end{gathered}
$$

This implies that $\mu_{\lambda_{C}}(v-r)=1, \mu_{\lambda_{C}}(v r)=1$ and $\gamma_{\lambda_{C}}(v-r)=0, \gamma_{\lambda_{C}}(v r)=0$. Thus $v-r$ and $v r \in C$. Hence $C=A \times B$ is a subring of $N R_{1} \times N R_{2}$.

Lemma 4.9. If $V=A \times B$ and $Q=C \times D$ are two subrings of $N R_{1} \times N R_{2}$ then their intersection $V \cap Q$ is also a subring of $N R_{1} \times N R_{2}$.

Theorem 4.10. Let $V=A \times B$ and $Q=C \times D$ be two intuitionistic fuzzy normed subrings of $N R_{1} \times N R_{2}$. Then $V \cap Q$ is subring of $N R_{1} \times N R_{2}$ if and only if the intuitionistic characteristic function $\lambda_{Z}=\left(\mu_{\lambda_{Z}}, \gamma_{\lambda_{Z}}\right)$ of $Z=V \cap Q$ is an intuitionistic fuzzy normed normal subring of $N R_{1} \times N R_{2}$.

Proof. Let $Z=V \cap Q$ be a subring of ring $N R_{1} \times N R_{2}$ and let $v=\left(v_{1}, v_{2}\right), r=\left(r_{1}, r_{2}\right) \in N R_{1} \times N R_{2}$. If $v, r \in Z=V \cap Q$, then by properties of intuitionistic characteristic function $\mu_{\lambda_{Z}}(v)=1=\mu_{\lambda_{Z}}(r)$ and $\gamma_{\lambda_{Z}}(v)=0=\gamma_{\lambda_{Z}}(r)$. Since $v-r$ and $v r \in Z$. Then, $\mu_{\lambda_{Z}}(v-r)=1=1 * 1=\mu_{\lambda_{Z}}(v) * \mu_{\lambda_{Z}}(r), \mu_{\lambda_{Z}}(v r)=1=$ 
$1 * 1=\mu_{\lambda_{Z}}(v) * \mu_{\lambda_{Z}}(r)$ and $\gamma_{\lambda_{Z}}(v-r)=0=0 \diamond 0=\gamma_{\lambda_{Z}}(v) \diamond \gamma_{\lambda_{Z}}(v), \gamma_{\lambda_{Z}}(v r)=0=0 \diamond 0=\gamma_{\lambda_{Z}}(v) \diamond \gamma_{\lambda_{Z}}(r)$. Therefore,

$$
\begin{aligned}
\mu_{\lambda_{Z}}(v-r) & \geq \mu_{\lambda_{Z}}(v) * \mu_{\lambda_{Z}}(r), \\
\mu_{\lambda_{Z}}(v r) & \geq \mu_{\lambda_{Z}}(v) * \mu_{\lambda_{Z}}(r), \\
\gamma_{\lambda_{Z}}(v-r) & \leq \gamma_{\lambda_{Z}}(v) \diamond \gamma_{\lambda_{Z}}(r), \\
\gamma_{\lambda_{Z}}(v r) & \leq \gamma_{\lambda_{Z}}(v) \diamond \gamma_{\lambda_{Z}}(r) .
\end{aligned}
$$

Since, $v r$ and $r v \in Z$, then $\mu_{\lambda_{Z}}(v r)=1=\mu_{\lambda_{Z}}(r v)$ and $\gamma_{\lambda_{Z}}(v r)=0=\gamma_{\lambda_{Z}}(r v)$ so $\mu_{\lambda_{Z}}(v r)=\mu_{\lambda_{Z}}(r v)$ and $\gamma_{\lambda_{Z}}(v r)=\gamma_{\lambda_{Z}}(r v)$. We also have when $v, r \notin Z$ :

$$
\begin{array}{ccc}
\mu_{\lambda_{C}}(v-r) \geq \mu_{\lambda_{C}}(v) * \mu_{\lambda_{C}}(r) & \text { and } & \mu_{\lambda_{C}}(v r) \geq \mu_{\lambda_{C}}(v) * \mu_{\lambda_{C}}(r), \\
\gamma_{\lambda_{C}}(v-r) \leq \gamma_{\lambda_{C}}(v) \diamond \gamma_{\lambda_{C}}(r) & \text { and } & \gamma_{\lambda_{C}}(v r) \leq \gamma_{\lambda_{C}}(v) \diamond \gamma_{\lambda_{C}}(r), \\
\mu_{\lambda_{C}}(v r)=\mu_{\lambda_{C}}(r v) & \text { and } & \gamma_{\lambda_{C}}(v r)=\gamma_{\lambda_{C}}(r v) .
\end{array}
$$

Hence the intuitionistic characteristic function $\lambda_{Z}=\left(\mu_{\lambda_{Z}}, \gamma_{\lambda_{Z}}\right)$ of $Z$ is an intuitionistic fuzzy normed normal subring of the ring $N R_{1} \times N R_{2}$.

Conversely, assume that the intuitionistic characteristic function $\lambda_{Z}=\left(\mu_{\lambda_{Z}}, \gamma_{\lambda_{Z}}\right)$ is an intuitionistic fuzzy normed normal subring. Let $v, r \in Z=V \cap Q$, then $\mu_{\lambda_{Z}}(v)=1=\mu_{\lambda_{Z}}(r)$ and $\gamma_{\lambda_{Z}}(v)=0=\gamma_{\lambda_{Z}}(r)$, hence:

$$
\begin{gathered}
\mu_{\lambda_{C}}(v-r) \quad \geq \mu_{\lambda_{C}}(v) * \mu_{\lambda_{C}}(r)=1 * 1=1, \\
\mu_{\lambda_{C}}(v r) \quad \geq \mu_{\lambda_{C}}(v) * \mu_{\lambda_{C}}(r)=1 * 1=1, \\
\gamma_{\lambda_{C}}(v-r) \quad \leq \gamma_{\lambda_{C}}(v) \diamond \gamma_{\lambda_{C}}(r) \quad=0 \diamond 0=0, \\
\gamma_{\lambda_{C}}(v r) \quad \leq \gamma_{\lambda_{C}}(v) \diamond \gamma_{\lambda_{C}}(r) \quad=0 \diamond 0=0 .
\end{gathered}
$$

Thus $\mu_{\lambda_{C}}(v-r)=1=\mu_{\lambda_{C}}(v r)$ and $\gamma_{\lambda_{C}}(v-r)=0=\gamma_{\lambda_{C}}(v r)$. This implies that $v-r$ and $v r \in Z$. Hence $Z$ is a subring of ring $N R_{1} \times N R_{2}$.

Proposition 4.11. If the IFS $A \times B$ is an intuitionistic fuzzy normed normal subring of the ring $N R_{1} \times N R_{2}$, then $\triangle A \times B=\left(\mu_{A \times B}, \mu_{A \times B}^{c}\right)$ is an intuitionistic fuzzy normed normal subring of the ring $N R_{1} \times N R_{2}$.

Proof. Let $A \times B$ be an intuitionistic fuzzy normed normal subring of $N R_{1} \times N R_{2}$ and let $(v, r),(z, d) \in$ $N R_{1} \times N R_{2}$. Then

$$
\begin{aligned}
\mu_{A \times B}^{c}((v, r)-(z, d)) & =1-\mu_{A \times B}((v, r)-(z, d)) \\
& \leq 1-\left(\mu_{A \times B}(v, r) * \mu_{A \times B}(z, d)\right) \\
& =1-\min \left\{\mu_{A \times B}(v, r), \mu_{A \times B}(z, d)\right\} \\
& =\max \left\{1-\mu_{A \times B}(v, r), 1-\mu_{A \times B}(z, d)\right\} \\
& =\max \left\{\mu_{A \times B}^{c}(v, r), \mu_{A \times B}^{c}(z, d)\right\} \\
& =\mu_{A \times B}^{c}(v, r) \diamond \mu_{A \times B}^{c}(z, d)
\end{aligned}
$$


and

$$
\begin{aligned}
\mu_{A \times B}^{c}((v, r) \circ(z, d)) & =1-\mu_{A \times B}((v, r) \circ(z, d)) \\
& \leq 1-\left(\mu_{A \times B}(v, r) * \mu_{A \times B}(z, d)\right) \\
& =1-\min \left\{\mu_{A \times B}(v, r), \mu_{A \times B}(z, d)\right\} \\
& =\max \left\{1-\mu_{A \times B}(v, r), 1-\mu_{A \times B}(z, d)\right\} \\
& =\max \left\{\mu_{A \times B}^{c}(v, r), \mu_{A \times B}^{c}(z, d)\right\} \\
& =\mu_{A \times B}^{c}(v, r) \diamond \mu_{A \times B}^{c}(z, d) .
\end{aligned}
$$

Thus $\triangle A \times B=\left(\mu_{A \times B}, \mu_{A \times B}^{c}\right)$ is an intuitionistic fuzzy normed subring $N R_{1} \times N R_{2}$.

$$
\begin{aligned}
\mu_{A \times B}^{c}((v, r) \circ(z, d)) & =1-\mu_{A \times B}((v, r) \circ(z, d)) \\
& =1-\mu_{A \times B}((z, d) \circ(v, r)) \\
& =\mu_{A \times B}^{c}((z, d) \circ(v, r))
\end{aligned}
$$

Hence, $\triangle A \times B=\left(\mu_{A \times B}, \mu_{A \times B}^{c}\right)$ is an intuitionistic fuzzy normed normal subring of $N R_{1} \times N R_{2}$.

Proposition 4.12. If the IFS $A \times B$ is an intuitionistic fuzzy normed normal subring of the ring $N R_{1} \times N R_{2}$, then $\diamond A \times B=\left(\gamma_{A \times B}^{c}, \gamma_{A \times B}\right)$ is an intuitionistic fuzzy normed normal subring of the ring $N R_{1} \times N R_{2}$.

Proof. Similar to the proof of Proposition 4.11

Corollary 4.13. An IFS $A \times B$ is an intuitionistic fuzzy normed normal subring of the ring $N R_{1} \times N R_{2}$ if and only if $\triangle A \times B=\left(\mu_{A \times B}, \mu_{A \times B}^{c}\right)$ (resp. $\left.\diamond A \times B=\left(\gamma_{A \times B}^{c}, \gamma_{A \times B}\right)\right)$ is an intuitionistic fuzzy normed normal subring of the ring $N R_{1} \times N R_{2}$.

Theorem 4.14. An IFS $A \times B$ is an intuitionistic fuzzy normed normal subring of the ring $N R_{1} \times N R_{2}$ if and only if the fuzzy subsets $\mu_{A \times B}$ and $\gamma_{A \times B}^{c}$ are intuitionistic fuzzy normed normal subring of the ring $N R_{1} \times N R_{2}$.

Proof. Let $A \times B=\left(\mu_{A \times B}, \gamma_{A \times B}\right)$ be an intuitionistic fuzzy normed normal subring of the ring $N R_{1} \times N R_{2}$. This implies that $\mu_{A \times B}$ is an intuitionistic fuzzy normed normal subring of $N R_{1} \times N R_{2}$. We have to show that $\gamma_{A \times B}^{c}$ is also an intuitionistic fuzzy normed normal subring of the ring $N R_{1} \times N R_{2}$. Let $(v, r),(z, d) \in$ $N R_{1} \times N R_{2}$. Then

$$
\begin{aligned}
\gamma_{A \times B}^{c}((v, r)-(z, d)) & =1-\gamma_{A \times B}((v, r)-(z, d)) \\
& \geq 1-\left(\gamma_{A \times B}(v, r) \diamond \gamma_{A \times B}(z, d)\right) \\
& =1-\max \left\{\gamma_{A \times B}(v, r), \gamma_{A \times B}(z, d)\right\} \\
& =\min \left\{1-\gamma_{A \times B}(v, r), 1-\gamma_{A \times B}(z, d)\right\} \\
& =\min \left\{\gamma_{A \times B}^{c}(v, r), \gamma_{A \times B}^{c}(z, d)\right\} \\
& =\gamma_{A \times B}^{c}(v, r) * \gamma_{A \times B}^{c}(z, d)
\end{aligned}
$$


and

$$
\begin{aligned}
\gamma_{A \times B}^{c}((v, r) \circ(z, d)) & =1-\gamma_{A \times B}((v, r)-(z, d)) \\
& \geq 1-\left(\gamma_{A \times B}(v, r) \diamond \gamma_{A \times B}(z, d)\right) \\
& =1-\max \left\{\gamma_{A \times B}(v, r), \gamma_{A \times B}(z, d)\right\} \\
& =\min \left\{1-\gamma_{A \times B}(v, r), 1-\gamma_{A \times B}(z, d)\right\} \\
& =\min \left\{\gamma_{A \times B}^{c}(v, r), \gamma_{A \times B}^{c}(z, d)\right\} \\
& =\gamma_{A \times B}^{c}(v, r) * \gamma_{A \times B}^{c}(z, d)
\end{aligned}
$$

Hence, $\gamma_{A \times B}^{c}$ is also an intuitionistic fuzzy normed subring of the ring $N R_{1} \times N R_{2}$.

$$
\begin{aligned}
\gamma_{A \times B}^{c}((v, r) \circ(z, d)) & =1-\gamma_{A \times B}((v, r) \circ(z, d)) \\
& =1-\gamma_{A \times B}((z, d) \circ(v, r)) \\
& =\gamma_{A \times B}^{c}((z, d) \circ(v, r)) .
\end{aligned}
$$

Hence, $\gamma_{A \times B}^{c}$ is an intuitionistic fuzzy normed normal subring of $N R_{1} \times N R_{2}$.

Conversely, suppose that $\mu_{A \times B}$ and $\gamma_{A \times B}^{c}$ are intuitionistic fuzzy normed normal subring of the ring $N R_{1} \times N R_{2}$. We have to show that $A \times B=\left(\mu_{A \times B}, \gamma_{A \times B}\right)$ is an intuitionistic fuzzy normed normal subring of the ring $N R_{1} \times N R_{2}$. Then

$$
\begin{aligned}
1-\gamma_{A \times B}((v, r)-(z, d)) & =\gamma_{A \times B}^{c}((v, r)-(z, d)) \\
& \geq \gamma_{A \times B}^{c}(z, d) * \gamma_{A \times B}^{c}(v, r) \\
& =\min \left\{\gamma_{A \times B}^{c}(z, d), \gamma_{A \times B}^{c}(v, r)\right\} \\
& =\min \left\{1-\gamma_{A \times B}(z, d), 1-\gamma_{A \times B}(v, r)\right\} \\
& =1-\max \left\{\gamma_{A \times B}(z, d), \gamma_{A \times B}(v, r)\right\} \\
& =1-\left(\gamma_{A \times B}(z, d) \diamond \gamma_{A \times B}(v, r)\right)
\end{aligned}
$$

and

$$
\begin{aligned}
1-\gamma_{A \times B}((v, r) \circ(z, d)) & =\gamma_{A \times B}^{c}((v, r) \circ(z, d)) \\
& \geq \gamma_{A \times B}^{c}(z, d) * \gamma_{A \times B}^{c}(v, r) \\
& =\min \left\{\gamma_{A \times B}^{c}(z, d), \gamma_{A \times B}^{c}(v, r)\right\} \\
& =\min \left\{1-\gamma_{A \times B}(z, d), 1-\gamma_{A \times B}(v, r)\right\} \\
& =1-\max \left\{\gamma_{A \times B}(z, d), \gamma_{A \times B}(v, r)\right\} \\
& =1-\left(\gamma_{A \times B}(z, d) \diamond \gamma_{A \times B}(v, r)\right) .
\end{aligned}
$$

Therefore, $A \times B=\left(\mu_{A \times B}, \gamma_{A \times B}\right)$ is an intuitionistic fuzzy normed subring of the ring $N R_{1} \times N R_{2}$.

$$
\begin{aligned}
1-\gamma_{A \times B}((v, r) \circ(z, d)) & =\gamma_{A \times B}^{c}((v, r) \circ(z, d)) \\
& =\gamma_{A \times B}^{c}((z, d) \circ(v, r)) \\
& =1-\gamma_{A \times B}((z, d) \circ(v, r)) .
\end{aligned}
$$


Therefore, $A \times B=\left(\mu_{A \times B}, \gamma_{A \times B}\right)$ is an intuitionistic fuzzy normed normal subring of the ring $N R_{1} \times$ $N R_{2}$.

Theorem 4.15. An IFS $A \times B$ is an intuitionistic fuzzy normed normal subring of the ring $N R_{1} \times N R_{2}$ if and only if the fuzzy subsets $\mu_{A \times B}^{c}$ and $\gamma_{A \times B}$ are intuitionistic fuzzy normed normal subring of the ring $N R_{1} \times N R_{2}$

Proof. Let $A \times B=\left(\mu_{A \times B}, \gamma_{A \times B}\right)$ be an intuitionistic fuzzy normed normal subring of the ring $N R_{1} \times N R_{2}$. This implies that $\gamma_{A \times B}$ is an intuitionistic fuzzy normed normal subring of $N R_{1} \times N R_{2}$. We have to show that $\mu_{A \times B}^{c}$ is also an an intuitionistic fuzzy normed normal subring of the $\operatorname{ring} N R_{1} \times N R_{2}$.

The proof of the first part is similar to the first part of Proposition 4.11.

Conversely, suppose that $\mu_{A \times B}^{c}$ and $\gamma_{A \times B}$ are intuitionistic fuzzy normed normal subring of the ring $N R_{1} \times$ $N R_{2}$. We have to show that $A \times B=\left(\mu_{A \times B}, \gamma_{A \times B}\right)$ is an intuitionistic fuzzy normed normal subring of the $\operatorname{ring} N R_{1} \times N R_{2}$. Then

$$
\begin{aligned}
& 1-\mu_{A \times B}((v, r)-(z, d)) \\
& =\mu_{A \times B}^{c}((v, r)-(z, d)) \\
& \leq \mu_{A \times B}^{c}(z, d) \diamond \mu_{A \times B}^{c}(v, r) \\
& =\max \left\{\mu_{A \times B}^{c}(z, d), \mu_{A \times B}^{c}(v, r)\right\} \\
& =\max \left\{1-\mu_{A \times B}(z, d), 1-\mu_{A \times B}(v, r)\right\} \\
& =1-\min \left\{\mu_{A \times B}(z, d), \mu_{A \times B}(v, r)\right\} \\
& =1-\left(\mu_{A \times B}(z, d) * \mu_{A \times B}(v, r)\right)
\end{aligned}
$$

and

$$
\begin{aligned}
& 1-\mu_{A \times B}((v, r) \circ(z, d)) \\
& =\mu_{A \times B}^{c}((v, r) \circ(z, d)) \\
& \leq \mu_{A \times B}^{c}(z, d) \diamond \mu_{A \times B}^{c}(v, r) \\
& =\max \left\{\mu_{A \times B}^{c}(z, d), \mu_{A \times B}^{c}(v, r)\right\} \\
& =\max \left\{1-\mu_{A \times B}(z, d), 1-\mu_{A \times B}(v, r)\right\} \\
& \left.=1-\min _{A \times B}\left(\mu_{A \times,}\right), \mu_{A \times B}(v, r)\right\} \\
& =1-\left(\mu_{A \times B}(z, d) * \mu_{A \times B}(v, r)\right) .
\end{aligned}
$$

Therefore, $A \times B=\left(\mu_{A \times B}, \gamma_{A \times B}\right)$ is an intuitionistic fuzzy normed subring of the ring $N R_{1} \times N R_{2}$.

$$
\begin{aligned}
1-\mu_{A \times B}((v, r) \circ(z, d)) & =\mu_{A \times B}^{c}((v, r) \circ(z, d)) \\
& =\mu_{A \times B}^{c}((z, d) \circ(v, r)) \\
& =1-\mu_{A \times B}((z, d) \circ(v, r)) .
\end{aligned}
$$

Therefore, $A \times B=\left(\mu_{A \times B}, \gamma_{A \times B}\right)$ is an intuitionistic fuzzy normed normal subring of the ring $N R_{1} \times$ $N R_{2}$. 


\section{Conclusion}

The objective of this paper was to initiate the notion of intuitionistic fuzzy normed normal subrings and to establish some relevant properties. We extended the notion of intuitionistic fuzzy normed subrings to intuitionistic fuzzy normed normal subrings. Also, we established the direct product of intuitionistic fuzzy normed normal subrings and examined some fundamental properties of direct product of intuitionistic fuzzy normed normal subrings. Further research could be done is to study the intuitionistic anti fuzzy normed normal subrings. We hope that in future, this concept would be a useful contribution to the study of intuitionistic fuzzy normed rings by generalizing other fundamental properties.

Conflicts of Interest: The author(s) declare that there are no conflicts of interest regarding the publication of this paper.

\section{REFERENCES}

[1] L.A. Zadeh, Fuzzy sets, Inform. Control. 8 (1965), 338-353.

[2] A. Rosenfeld, Fuzzy groups, J. Math. Anal. Appl. 35 (1971), 512-517.

[3] W. Wu, Normal fuzzy subgroups, Fuzzy Math. 1 (1981), 21-30.

[4] W. Liu, Fuzzy invariant subgroups and fuzzy ideals, Fuzzy Sets Syst. 8 (1982), 133-139.

[5] N. Mukherjee, Fuzzy normal subgroups and fuzzy cosets, Inform. Sci. 34 (1984), 225-239.

[6] W. Wu, Fuzzy congruences and normal fuzzy subgroups, Math. Appl. 1 (1988), 9-20.

[7] M. Mishref, Normal fuzzy subgroups and fuzzy normal series of finite groups, Fuzzy Sets Syst. 72 (1995), $379-383$.

[8] S. Abdullah, M. Aslam, T. A. Khan, M. Naeem, A new type of fuzzy normal subgroups and fuzzy cosets, J. Intell. Fuzzy Syst. 25 (2013), 37-47.

[9] K.T. Atanassov, Intuitionistic fuzzy sets, In Intuitionistic Fuzzy Sets, Springer, Berlin, Germany, 1999, 1-137.

[10] K. Hur, S.Y. Jang, H.W. Kang, Intuitionistic fuzzy normal subgroups and intuitionistic fuzzy cosets, Honam Math. J. 26 (2004), 559-587.

[11] M.F. Marashdeh, A.R. Salleh, The intuitionistic fuzzy normal subgroup, Int. J. Fuzzy Log. Intell. Syst. 10 (2010), 82-88.

[12] V. Veeramani, K. Arjunan, N. Palaniappan, Some properties of intuitionistic fuzzy normal subrings, Appl. Math. Sci. 4 (2010), 2119-2124.

[13] P. Sharma, On Intuitionistic fuzzy magnified translation in rings, Int. J. Algebra. 1 (2011), 1451-1458.

[14] N. Abed Alhaleem, A.G. Ahmad, Intuitionistic Fuzzy Normed Subrings and Intuitionistic Fuzzy Normed Ideals, Mathematics. 8 (2020), 1594.

[15] A. Emniyet, M. Şahin, Fuzzy normed rings, Symmetry. 10 (2018), 515.

[16] R. Arens, A generalization of normed rings, Pac. J. Math. 2 (1952), 455-471.

[17] M.A. Najmark, Normed rings, Noordhoff, Groningen, The Netherlands, 1964.

[18] I. Gelfand, D. Raikov, G. Shilov, Commutative Normed Rings, American Mathematical Society, USA, 2002.

[19] A.U Alkouri, A.R. Salleh, Complex Atanassov's intuitionistic fuzzy relation, Abstr. Appl. Anal. 2013 (2013), 287382.

[20] M.O. Alsarahead, A.G. Ahmad, Complex intuitionistic fuzzy ideals, AIP Conf. Proc. 1940 (2018), 020118.

[21] P. Sharma, G. Kaur, On intuitionistic fuzzy prime submodules, Notes IFS. 24 (2018), 97-112. 
[22] A. Al-Masarwah, A.G. Ahmad, Structures on Doubt Neutrosophic Ideals of BCK/BCI-Algebras under (s, t)-Norms, Neutrosophic Sets Syst. 33 (2020), 17.

[23] M. Gupta, J. Qi, Theory of t-norms and fuzzy inference methods, Fuzzy Sets Syst. 40 (1991), 431-450. 